\title{
X-Ray Selected Type 2 QSOs and Their Host Galaxies
}

\author{
Vincenzo Mainieri ${ }^{1}$ and the COSMOS Collaboration \\ ${ }^{1}$ ESO, Karl-Schwarschild-Strasse 2, D-85748 Garching bei München, Germany \\ Email: vmainier@eso.org
}

\begin{abstract}
We present a large sample of X-ray selected type 2 QSOs from the $X M M$-COSMOS survey. Type 2 QSOs are luminous AGN whose central engines are obscured by large amounts of gas and dust. The selection criteria we have used are based on high X-ray luminosity $\left(L_{\mathrm{X}}>\right.$ $\left.10^{44} \mathrm{erg} \mathrm{s}^{-1}\right)$ and heavy obscuration $\left(N_{\mathrm{H}}>10^{22} \mathrm{~cm}^{-2}\right)$. We derived stellar masses and starformation rate estimates for the host galaxies from the best fit of the observed photometry. Type 2 QSOs are generally hosted in massive galaxies with on-going star formation.
\end{abstract}

Keywords. galaxies: active, quasars: general, X-rays: general

\section{Introduction}

Although the fraction of obscured AGN is found in several studies to decrease with luminosity, a non-negligible population of obscured QSOs is still required by X-ray background synthesis models. Radio-loud type 2 QSOs have been known for a long time thanks to radio surveys (see McCarthy 1993 for a comprehensive review), while radioquiet type 2 QSOs have been observed only recently in Chandra and XMM-Newton X-ray surveys (Dawson et al. 2001; Norman et al. 2002; Mainieri et al. 2002; Stern et al. 2002; Della Ceca et al. 2003; Fiore et al. 2003; Tozzi et al. 2006) and optical surveys (SDSS, Zakamska et al. 2003).

\section{Sample Selection}

Our selection of type 2 QSOs is based only on X-ray properties: we selected sources with de-absorbed $L_{\mathrm{X}}(0.5-10 \mathrm{keV})>10^{44} \mathrm{erg} \mathrm{s}^{-1}$ and column densities $N_{\mathrm{H}}>10^{22} \mathrm{~cm}^{-2}$. Both X-ray luminosities and obscuration have been derived from a detailed X-ray spectral analysis of the $\sim 1800$ point-like sources of the XMM-COSMOS survey (Cappelluti et al. 2009). For a detailed description of the X-ray spectral procedure we refer the reader to Mainieri et al. (2007). We recall here only that we used a set of models including a simple power law, a power law modified by intrinsic absorption at the redshift of the source, and a pure reflection model (pexrav). Additional components would have to be included to describe the presence of a soft excess or of the Fe $\mathrm{K} \alpha$ emission line.

We currently have a sample of X-ray selected type 2 QSOs consisting of 157 objects. We have cross-correlated this sample with the output of ongoing spectroscopic campaings in the COSMOS field (Lilly et al. 2009; Trump et al. 2009): 40\% of the type 2 QSOs have a spectroscopic redshift. For the remaining $60 \%$ of the sample, we use the very accurate photometric redshifts available for the AGN in the COSMOS field (Salvato et al. 2009). Our X-ray selected sample of type 2 QSOs is complementary to the SDSS optically selected sample presented by Zakamska et al. (2003) in terms of redshift range covered: the SDSS sample based on [O III] luminosities cannot be at redshift higher than $z \approx 0.8$, while our sample is almost entirely at $z>0.8$. 


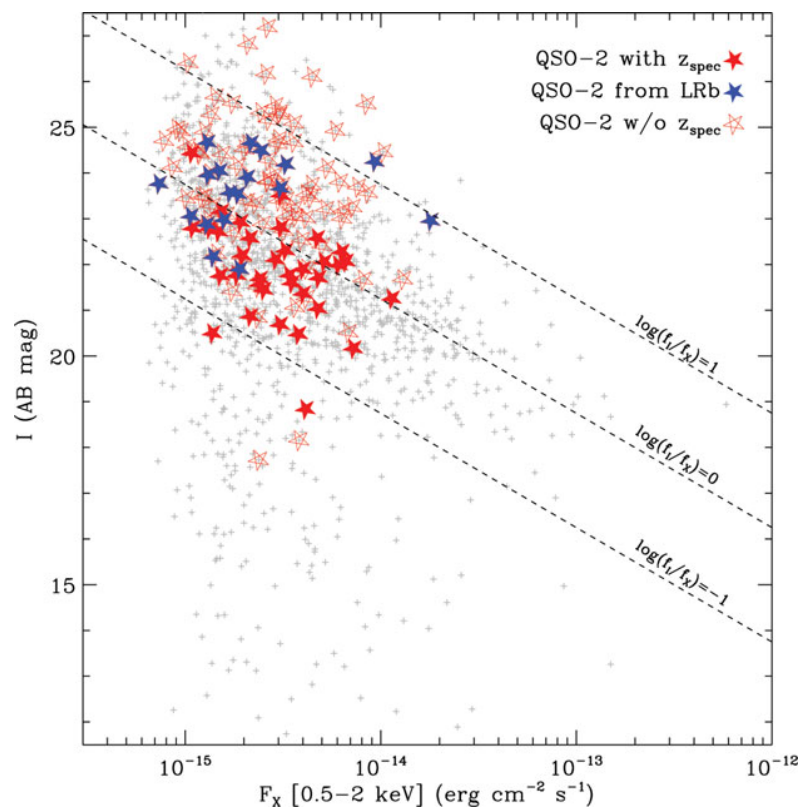

Figure 1. X-ray flux in the $0.5-2 \mathrm{keV}$ band versus the $I$-band magnitude of the selected counterpart (Brusa et al. 2010). The crosses are the $\sim 1800 \mathrm{X}$-ray point-like sources in the $X M M-$ COSMOS survey. The filled (empty) stars are type 2 QSOs for which a spectroscopic (photometric) redshift is available.

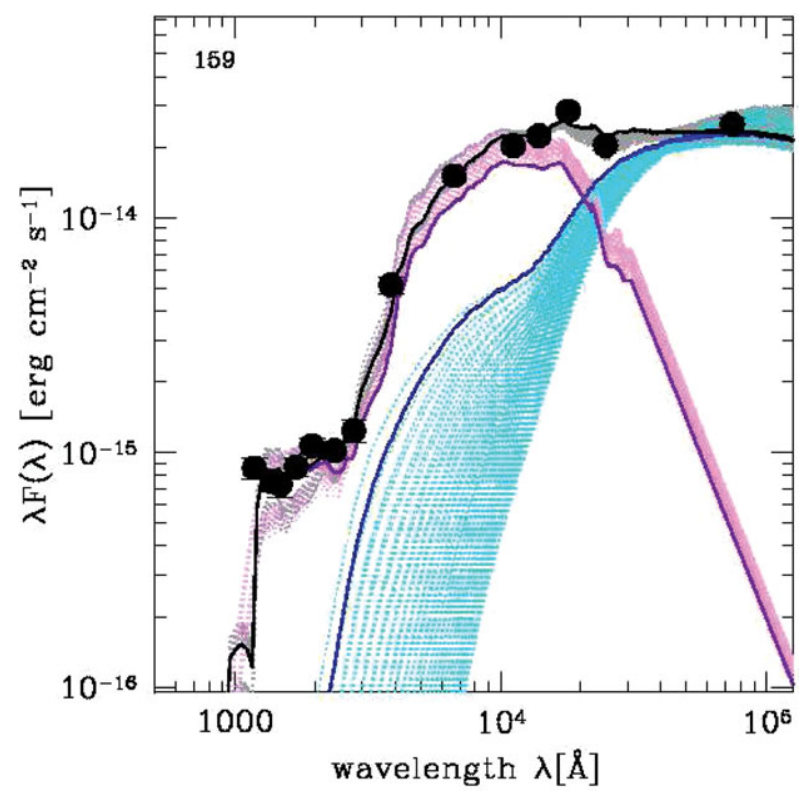

Figure 2. An example of SED decompositions. Black circles are the observed photometric measurements in the rest-frame. Heavy lines correspond respectively to the galaxy and AGN template found as the best fit solution. 


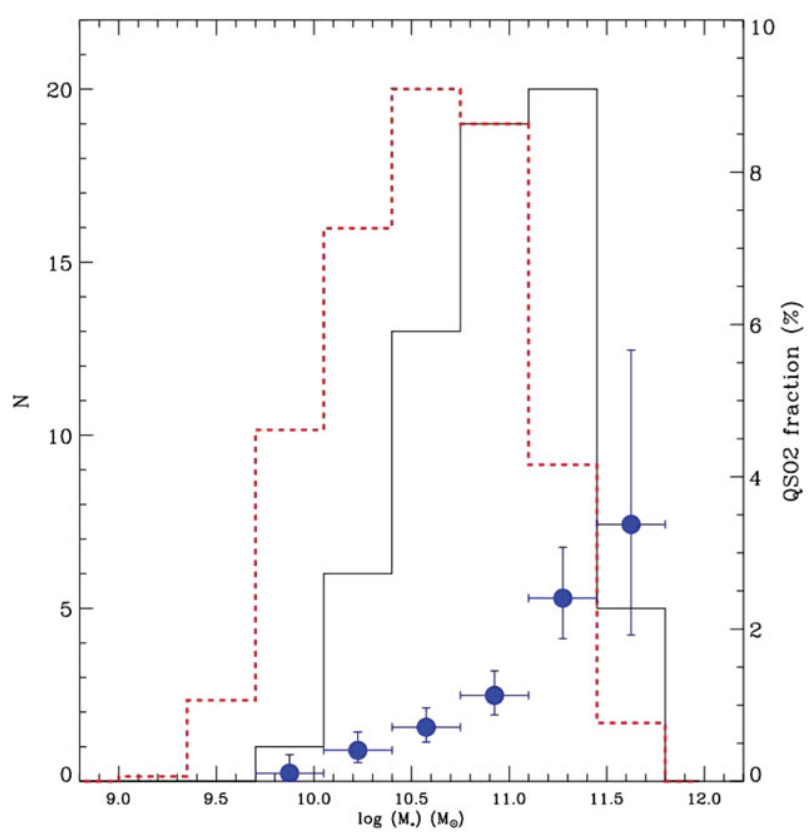

Figure 3. Stellar mass distribution of the type 2 QSO host galaxies (solid histogram) and of the galaxies' parent sample (dashed histogram). The data points represent the fraction of galaxies hosting type 2 QSOs in bins of stellar masses.

\section{Host-Galaxy Properties}

We now want to study the properties of the galaxies where the type 2 QSOs are hosted. In particular, we would like to derive estimates for the stellar mass and star formation rate (SFR) of these host galaxies. We have used the impressive multiwavelength coverage of the COSMOS field to derive host-galaxy properties through detailed model fitting of the total SED of the type 2 QSOs. We fit the observed SED with a grid of models made from a combination of AGN and host galaxy templates (see Merloni et al. 2009). The combination of templates allows us to disentangle the emission of the host galaxy from the contribution of the central black hole. We show in Figure 2 an example of this composite fit. From the best-fit SED, we use the galaxy component to estimate both stellar mass and SFR of the host.

\subsection{Stellar Mass}

We plot in Figure 3 the fraction of galaxies hosting a type 2 QSOs as a function of their stellar masses. In order to derive this fraction we have selected a parent sample of galaxies from the COSMOS field covering the same redshift range of our type 2 QSOs sample. The parent sample include $\sim 7000$ galaxies for which we have derived stellar mass estimates using the SED-fitting technique described above. We follow the technique discussed in $\S 3.1$ of Lehmer et al. (2007) to determine the type 2 QSOs fraction that accounts for the spatially varying sensitivity limits of the $X M M$ observations of the COSMOS field. From Figure 3 we see that the fraction of galaxies hosting type 2 QSOs monotonically increases with stellar masses. This is similar to what has already been found for the overall AGN population (Kauffmann et al. 2003; Best et al. 2005; Silverman et al. 2009). 


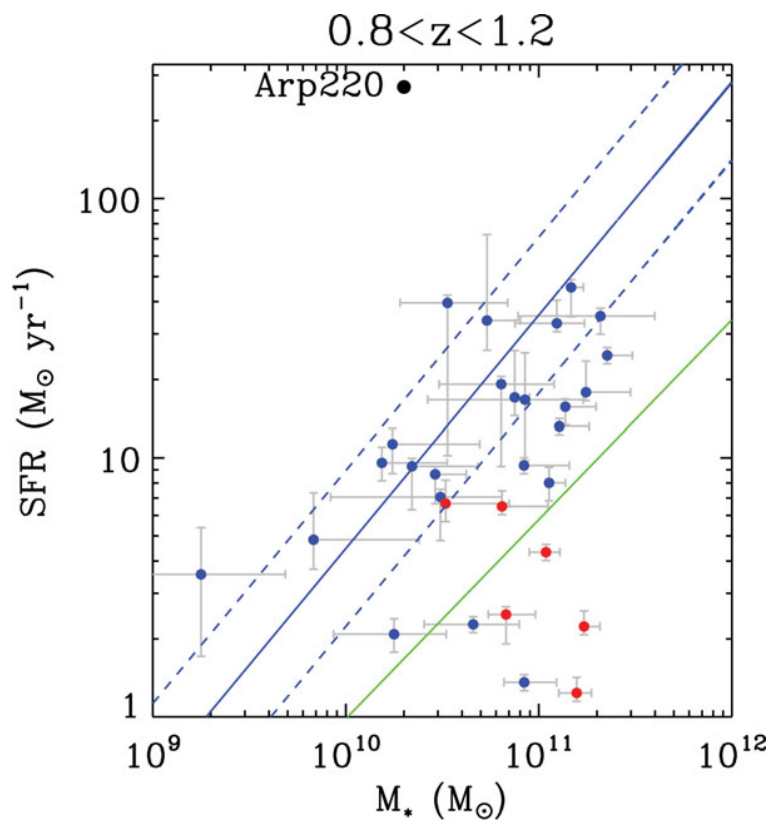

Figure 4. SFR versus stellar masses for the host galaxies of the type 2 QSOs (filled circles). The continuum lines are: (Left:) the relationship found for $z=0.8-1.2$ star-forming galaxies by Elbaz et al. (2007); (Right:) the correlation found for local galaxies with no AGN contribution in the SDSS (Brinchmann et al. 2004).

\section{2. $S F R$}

It has been recently observed that for blue star-forming galaxies there is a tight correlation between SFR and stellar mass (e.g., Noeske et al. 2007; Elbaz et al. 2007). In this plane, galaxies are generally well-separated in two clouds: one at high stellar masses and weak SFR and one with a continuous increase of the SFR with stellar mass. In Figure 4 we compare the host galaxies of our type 2 QSOs with the correlation found for blue starforming galaxies at $z \approx 1$. If we exclude the type 2 QSOs with red $(U-B)$ rest-frame colors that tend to cluster in the bottom-right corner of the plot, the majority of our type 2 QSOs have SFR levels consistent with that expected for star-forming galaxies. This is also consistent with the morphology of the type 2 QSOs host galaxies: the majority are spiral galaxies.

\section{References}

Best, P. N., et al. 2005, MNRAS, 362, 25

Brinchmann, J., et al. 2004, MNRAS, 351, 1151

Brusa, M. et al. 2010, submitted to $A \mathscr{E} A$

Cappelluti, N., et al. 2009, A\&A A, 497, 635

Dawson, S., et al. 2001, AJ, 122, 598

Della Ceca, R., et al. 2003, A\&A A, 406, 555

Elbaz, D., et al. 2007, A\&SA, 468, 33

Fiore, F., et al. 2003, $A \& A, 409,79$

Kauffmann, G., et al. 2003, MNRAS, 346, 1055

Lehmer, B. D., et al. 2007, ApJ, 657, 681

Lilly, S. J., et al. 2009, ApJS, 184, 218

Mainieri, V., et al. 2002, A\&A A, 393, 425 
Mainieri, V., et al. 2007, ApJS, 179, 95

McCarthy, P. J. 1993, ARAEA, 31, 639

Merloni, A., et al. 2009, ApJ, in press [arXiv:0910.4970]

Noeske, K. G., et al. 2007, ApJ, 660, 43

Norman, C., et al. 2002, ApJ, 571, 218

Salvato, M., et al. 2009, ApJ, 690, 1250

Silverman, J. D., et al. 2009, ApJ, 696, 396

Stern, D., et al. 2002, ApJ, 568, 71

Tozzi, P., et al. 2006, A\& $A, 451,457$

Trump, J. R., et al. 2009, ApJ, 696, 1195

Zakamska, N. L., et al. 2003, AJ, 126, 2125 